Forum $2021 \cdot 36: 417$

https://doi.org/10.1007/s12312-021-00976-3

Online publiziert: 3. September 2021

(C) Springer Medizin Verlag $\mathrm{GmbH}$, ein Teil von

Springer Nature 2021

\section{German Cancer Survivors Week 2021 - Neues Konzept - hohe Reichweite}

"Leben mit Krebs", das ist oftmals eine Herausforderung für die Betroffenen. Denn selbst wenn die Behandlung abgeschlossen ist, sehen sich krebskranke Menschen oft mit gesundheitlichen Einschränkungen konfrontiert, die sich auf ihre gesamte Lebenssituation auswirken: auf Familie, Freundeskreis und Beruf. Welche Möglichkeiten gibt es, um die eigene Lebensqualität zu verbessern und am Leben teilhaben zu können? Der German Cancer Survivors Day der Deutschen Krebsstiftung wurde ins Leben gerufen, um die Öffentlichkeit auf die Herausforderungen von Krebspatient*innen aufmerksam zu machen und den Erfahrungsaustausch unter den Betroffenen zu fördern. Bislang als Präsenzveranstaltung in Berlin durchgeführt, fand die Veranstaltung 2021 erstmals digital statt. Dabei wurde der German Cancer Survivors Day zur German Cancer Survivors Week (GCSW) - einer Woche für Krebsbetroffene. Im Zeitraum vom 30. Mai bis zum 5. Juni konnten Interessierte täglich an einer anderen Informationsveranstaltung oder interaktiven Fragerunde teilnehmen. Das abwechslungsreiche Programm wurde von der Deutschen Krebsstiftung gemeinsam mit den sechs Landeskrebsgesellschaften aus Baden-Württemberg, Brandenburg, Hamburg, Nordrhein-Westfalen, Sachsen und Thüringen gestaltet.

„Die COVID-19-Pandemie zwang uns dazu, vom Format der Präsenzveranstaltung abzuweichen", erklärte Iris Meumann von der Deutschen Krebsstiftung. Denn an ein Treffen auf dem belebten Potsdamer Platz mitten in Berlin, wie in den vergangenen Jahren,

\section{Infobox 1}

\section{GCSW 2021 im Mitschnitt}

Alle Beiträge zur German Cancer Survivors Week 2021 finden Sie unter diesem Link: www.deutsche-krebsstiftung.de/projects/german-cancer-survivors-week-2021/ war in diesem Jahr nicht zu denken. So entstand ein Konzept für insgesamt sechs zweistündige Online-Sitzungen, die werktags ab 17.00 Uhr oder am Samstagvormittag gesendet wurden, entweder als vorproduzierte Videos oder im Live-Stream. Auf dem Programm standen Fragen wie: Wie lässt sich Komplementäre Medizin als Ergänzung zur Schulmedizin einsetzen? Was tun gegen krebsbedingte Fatigue? Was bringen Achtsamkeitsübungen und Sport bei Krebs? Wie lässt sich die Kommunikation mit dem Umfeld verbessern? Was, wenn die Krankheit zu finanzieller Not führt? Ein Poetry-Slam, ein Kochvideo, Interviews und Podiumsdiskussionen rundeten das Programm ab. „Unsere anfängliche Befürchtung, der Kontakt zu den Nutzer*innen sei online schwer herzustellen, hat sich nicht bewahrheitet. Wer wollte, konnte im Chat Fragen an die Expert*innen stellen und die Teilnehmer*innen haben diese Mög-
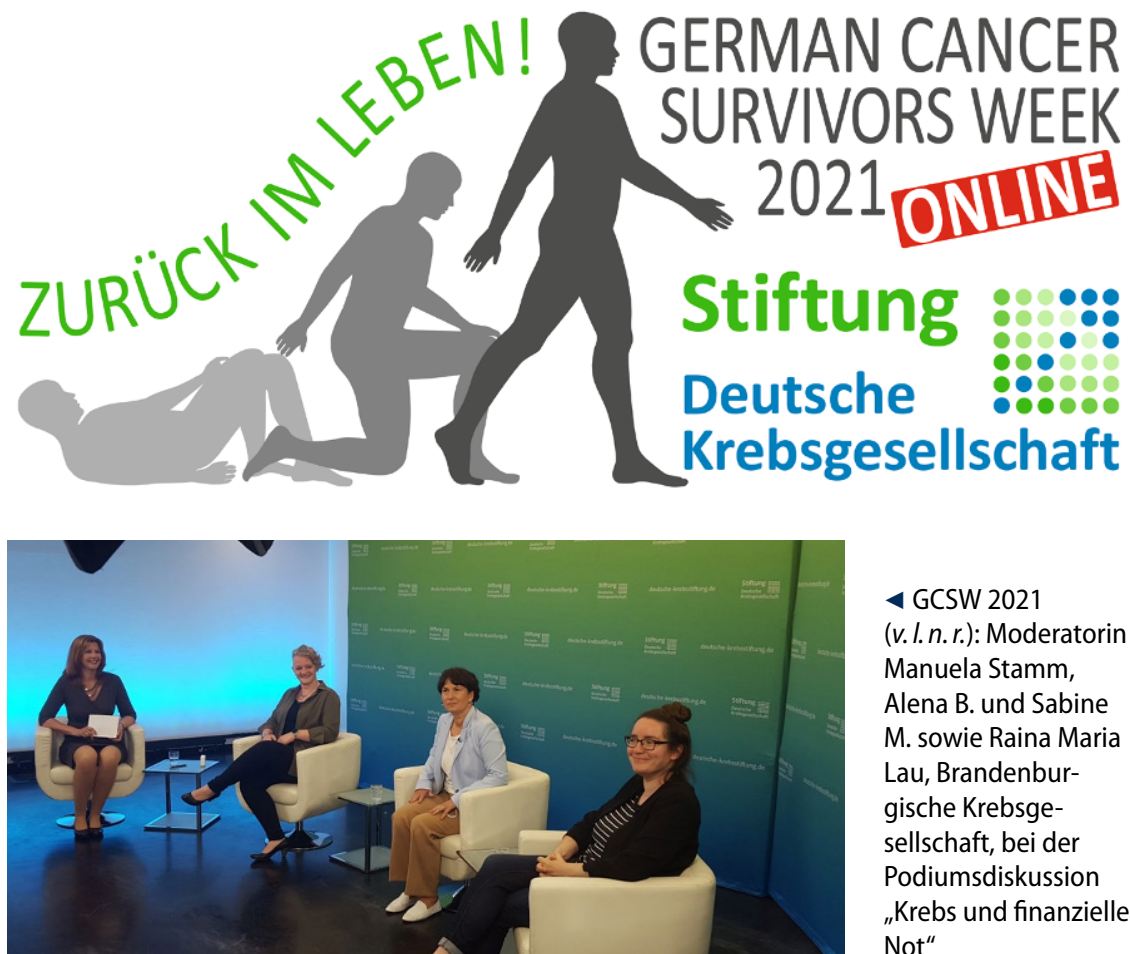

lichkeit auch wirklich zahlreich genutzt", freute sich Astrid Heßmer, Geschäftsführerin der Thüringischen Krebsgesellschaft. Die Reichweiten sprechen für sich, insgesamt wurden die Beiträge bislang ungefähr 1200-mal aufgerufen, entweder direkt oder nachträglich.,Das Online-Format und die Bündelung der Kräfte aus mehreren Landeskrebsgesellschaften haben sich bewährt - diese positive Erfahrung nehmen wir gerne in die Vorbereitung der Veranstaltung im nächsten Jahr mit", so das Fazit von Iris Meumann.

\section{Korrespondenzadresse}

\section{Katrin Mugele}

Deutsche Krebsgesellschaft e.V. Kuno-Fischer-Str. 8, Berlin, Deutschland mugele@krebsgesellschaft.de
4 GCSW 2021

(v. I.n.r.): Moderatorin Manuela Stamm, Alena B. und Sabine M. sowie Raina Maria Lau, Brandenburgische Krebsgesellschaft, bei der Podiumsdiskussion „Krebs und finanzielle Not" 